\title{
Temperature-Dependent Modulation of Excitatory Transmission in Hippocampal Slices Is Mediated by Extracellular Adenosine
}

\author{
Susan A. Masino ${ }^{1,2}$ and Thomas V. Dunwiddie $1,2,3$

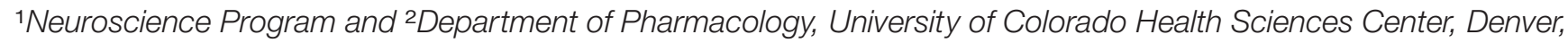 \\ Colorado 80262, and 3Veterans Affairs Medical Research Service, Denver, Colorado 80220
}

\begin{abstract}
Although extracellular adenosine concentrations in brain are increased markedly by a variety of stimuli such as hypoxia and ischemia, it has been difficult to demonstrate large increases in adenosine with stimuli that do not result in pathological tissue damage. The present studies demonstrate that increasing the temperature at which rat hippocampal brain slices are maintained (typically from 32.5 to $38.5^{\circ} \mathrm{C}$ ) markedly inhibits excitatory synaptic transmission. This effect was reversible on cooling, readily repeatable, and was blocked by $A_{1}$ receptor antagonists and by adenosine deaminase, suggesting that it was mediated by increased activation of presynaptic adenosine $A_{1}$ receptors by endogenous adenosine. This increase in adenosinergic inhibition was not a response to hyperthermia per se, because it could be elicited by temperatures that remained
\end{abstract}

Low concentrations of adenosine normally are found in the extracellular space of brain, concentrations that are sufficient to occupy, at least partially, the relatively high-affinity $A_{1}$ and $A_{2 A}$ receptors even under basal conditions $\left[\mathrm{A}_{1}\right.$ (Dunwiddie and Hoffer, 1980; Bruns et al., 1986; Dunwiddie and Diao, 1994); $\mathrm{A}_{2 \mathrm{~A}}$ (Cunha et al., 1996; Svenningsson et al., 1997)]. Activation of $A_{1}$ receptors exerts a tonic influence on electrophysiological activity in regions such as the hippocampus (Dunwiddie, 1985), olfactory cortex (Motley and Collins, 1983), and striatum (Flagmeyer et al., 1997), where adenosine acts primarily on presynaptic glutamatergic receptors to inhibit excitatory synaptic transmission. However, a variety of situations can increase extracellular adenosine levels dramatically and virtually abolish excitatory synaptic transmission. Most of the conditions known to promote such large increases in extracellular adenosine are those in which the supply of oxygen and energy substrates is not adequate to meet the energy demands of the brain, such as hypoxia (Rubio et al., 1975; Fowler, 1993; Zhu and Krnjević, 1993), ischemia (Berne et al., 1974), metabolic inhibition (Zhu and Krnjević, 1997), or electrical hyperactivity (Rubio et al., 1975; Lloyd et al., 1993). All of these conditions ultimately can become pathological and result in significant cell loss. Although these stressful conditions that increase extracellular adenosine are physiologically relevant, they are also relatively unusual. However, smaller changes in brain metabolic balance or other variables occur on a regular basis, and it is

Received Sept. 14, 1998; revised Dec. 15, 1998; accepted Dec. 22, 1998.

This work was supported by Grant R01 NS 29173 from the National Institute of Neurological Disorders and Stroke and the Veterans Administration Medical Research Service. We acknowledge the contribution of Daniel Lopez.

Correspondence should be addressed to Dr. Susan A. Masino, Neuroscience Program, University of Colorado Health Sciences Center, Neuroscience B138, 4200 East Ninth Avenue, Denver, CO 80262.

Copyright (C) 1999 Society for Neuroscience $\quad 0270-6474 / 99 / 191932-08 \$ 05.00 / 0$ entirely within the hypothermic range (e.g., from 32.5 to $35.5^{\circ} \mathrm{C}$ ). The increased activity at $A_{1}$ receptors appeared to be attributable to the direct release of adenosine via nucleoside transporters; the release of adenine nucleotides, linked to either the activation of NMDA receptors or the increased efflux of cAMP, appeared not to be involved. These results suggest that changes in brain temperature can alter the regulation of extracellular adenosine in rat brain slices and that increased adenosine release may be an important regulatory mechanism for countering increased excitability consequent to increased brain temperature.

Key words: adenosine; hippocampus; temperature; hyperthermia; $A_{1}$ receptors; CA1

possible that more modest physiological changes transiently regulate adenosine levels and neuronal excitability in a more subtle manner.

A common aspect of many stimuli that release adenosine from brain slices is a reduction in the ability of cells to synthesize ATP (e.g., by removing glucose or oxygen from the superfusion buffer) (Fowler, 1993; Zhu and Krnjević, 1993, 1997). Another manipulation that might alter metabolic balance and be physiologically stressful is changing the temperature. In general, increases in temperature correlate with an increased metabolic rate, whereas decreases in temperature result in reductions in the metabolic rate; hypothermia is a well known mechanism of cerebroprotection, both in intact animals (Barone et al., 1997) and in brain slices (Tanimoto and Okada, 1987). In vivo, small fluctuations in temperature occur throughout the day (Refinetti and Menaker, 1992), and larger changes can be initiated by physical exercise, heat stress, trauma, or illness (Anderson et al., 1983; Kluger, 1991; Rothwell, 1994). In addition, the function of the CNS is known to be particularly sensitive to increased temperature (Anderson et al., 1983). Temperature is usually a well controlled experimental variable in in vitro studies and an important physiological one, but its direct effects have not been characterized extensively in brain slices, particularly insofar as its relationship with endogenous adenosine. To this end we have characterized the effects of moderate, transient temperature increases on evoked CA1 field potentials in the hippocampal slice and examined the extent to which these effects can be attributed to changes in endogenous adenosine.

\section{MATERIALS AND METHODS}

Slice preparation. Transverse hippocampal slices were obtained from 6- to 8-week-old Sprague Dawley rats by using standard procedures (Dunwiddie and Lynch, 1978; Dunwiddie and Hoffer, 1980). Briefly, after decap- 
itation into ice-cold artificial CSF (aCSF), $400 \mu \mathrm{m}$ slices were made on a Sorvall TC-2 tissue chopper and incubated either at room temperature $\left(\sim 23^{\circ} \mathrm{C}\right)$ or at $32.5^{\circ} \mathrm{C}$. The aCSF used for dissection, incubation, and submerged, perfused recordings $(2.0 \mathrm{ml} / \mathrm{min}$ ) contained (in $\mathrm{mM}): \mathrm{NaCl}$ 126.0, $\mathrm{KCl} 3.0, \mathrm{MgSO}_{4} 1.5$, D-glucose $11, \mathrm{CaCl}_{2} 2.4, \mathrm{NaH}_{2} \mathrm{PO}_{4} 1.2$, and $\mathrm{NaHCO}_{3} 25.9$ that was bubbled continuously with a $95 \% \mathrm{O}_{2} / 5 \% \mathrm{CO}_{2}$ mixture. Slices were incubated undisturbed for $90 \mathrm{~min}$ before electrophysiological recording.

Electrophysiological recording. Slices were placed on a nylon net in the recording chamber and superfused continuously $(2.0 \mathrm{ml} / \mathrm{min})$ with aCSF bubbled with $95 \% \mathrm{O}_{2} / 5 \% \mathrm{CO}_{2}$. Extracellular field EPSPs (fEPSPs) were recorded from the CA1 region of the stratum radiatum by using glass micropipettes (10-15 M $\Omega$ ) filled with $3 \mathrm{M} \mathrm{NaCl}$. A twisted bipolar stimulating electrode was placed to stimulate the Schaffer collaterals in stratum radiatum every $30 \mathrm{sec}$. Stimulation intensity was adjusted such that the fEPSP was between 0.5 and $1.2 \mathrm{mV}$. Data were recorded via an $\mathrm{AC}$ amplifier and digitized and stored in the computer for later analysis.

Temperature manipulation. After a 5-10 min stable baseline was established, the temperature of the superfusion medium was changed slowly $\left(\sim 1.0^{\circ} \mathrm{C} / \mathrm{min}\right.$; see Fig. $\left.1 \mathrm{~A}\right)$ from the baseline recording temperature to either $32.5^{\circ} \mathrm{C}$ (slices starting from a room temperature baseline) or $38.5^{\circ} \mathrm{C}$ (slices starting from a $32.5^{\circ} \mathrm{C}$ baseline). The temperature was regulated with a thermostatic controller to within $0.5^{\circ} \mathrm{C}$, and the chamber temperature was measured with a small thermistor (YSI 511, Yellow Springs Instruments, Yellow Springs, $\mathrm{OH}$ ) placed directly in the recording chamber along with the tissue slice. In the majority of experiments the incubation and baseline fEPSP measurements were made at $32.5^{\circ} \mathrm{C}$; the higher temperature $\left(38.5^{\circ} \mathrm{C}\right)$ was achieved, was maintained for 5-7 min, and gradually was decreased back to baseline at a rate similar to that of the previous increase. Unless noted otherwise, this initial test protocol was used to characterize the temperature sensitivity of each slice, and this was compared with the temperature sensitivity of the same slice after a physiological or pharmacological manipulation. In a subset of experiments the higher temperature was maintained for $>40$ and up to $65 \mathrm{~min}$.

Drug application. A baseline fEPSP was reestablished after the initial period of increased temperature to evaluate the degree of recovery, after which any drugs that were applied were superfused for a minimum of 12 min before the temperature again was increased. Thus, the effect of the temperature changes on the fEPSP was compared before (control) and after drug treatment within each hippocampal slice.

Analysis. Statistical analyses included linear regression analysis and Student's two-tailed paired $t$ tests.

Chemicals. Adenosine deaminase (type VI), DL-2-amino-5-phosphonopentanoic acid (APV), $\alpha, \beta$-methyleneadenosine 5 '-diphosphate (AOPCP), dipyridamole, guanosine $5^{\prime}$-monophosphate (GMP), probenecid, theophylline, and all aCSF constituents were obtained from Sigma (St. Louis, MO); 8-cyclopentyl-1, 3-dipropylxanthine (DPCPX), dizocilpine maleate (MK-801), and 4-[(3-butoxy-4-methoxyphenyl)methyl]2-imidazolidinone (Ro 20-1724) were obtained from Research Biochemicals (Natick, MA). FPL 67156 was a gift from Fisons Pharmaceuticals (Leicestershire, UK).

\section{RESULTS}

\section{Temperature increases inhibit excitatory synaptic responses}

In initial experiments we observed that increasing the temperature of hippocampal slices from the usual recording temperature of $32.5 \rightarrow 38.5^{\circ} \mathrm{C}$ resulted in a profound depression in the amplitude of the fEPSP recorded in CA1. This decrease was sustained for the duration of the increased temperature and was completely reversible when the temperature was returned to $32.5^{\circ} \mathrm{C}$ (Fig. $1 \mathrm{~A}$ ). When the same slice was challenged with a second increase in temperature, the second response was typically of the same magnitude as the initial response (Fig. $1 B$ ). In six slices tested in this manner there was no significant difference between the amount of inhibition produced by the initial versus a subsequent temperature increase $[72.9 \pm 6.6 \%$ vs $73.8 \pm 7.8 \%$; not significant (n.s.); paired $t$ test]. Thus, in subsequent experiments each slice was used as its own control to examine the effects of experimental manipulations on the fEPSP response. There was considerable variability in the amount of inhibition exhibited by individual
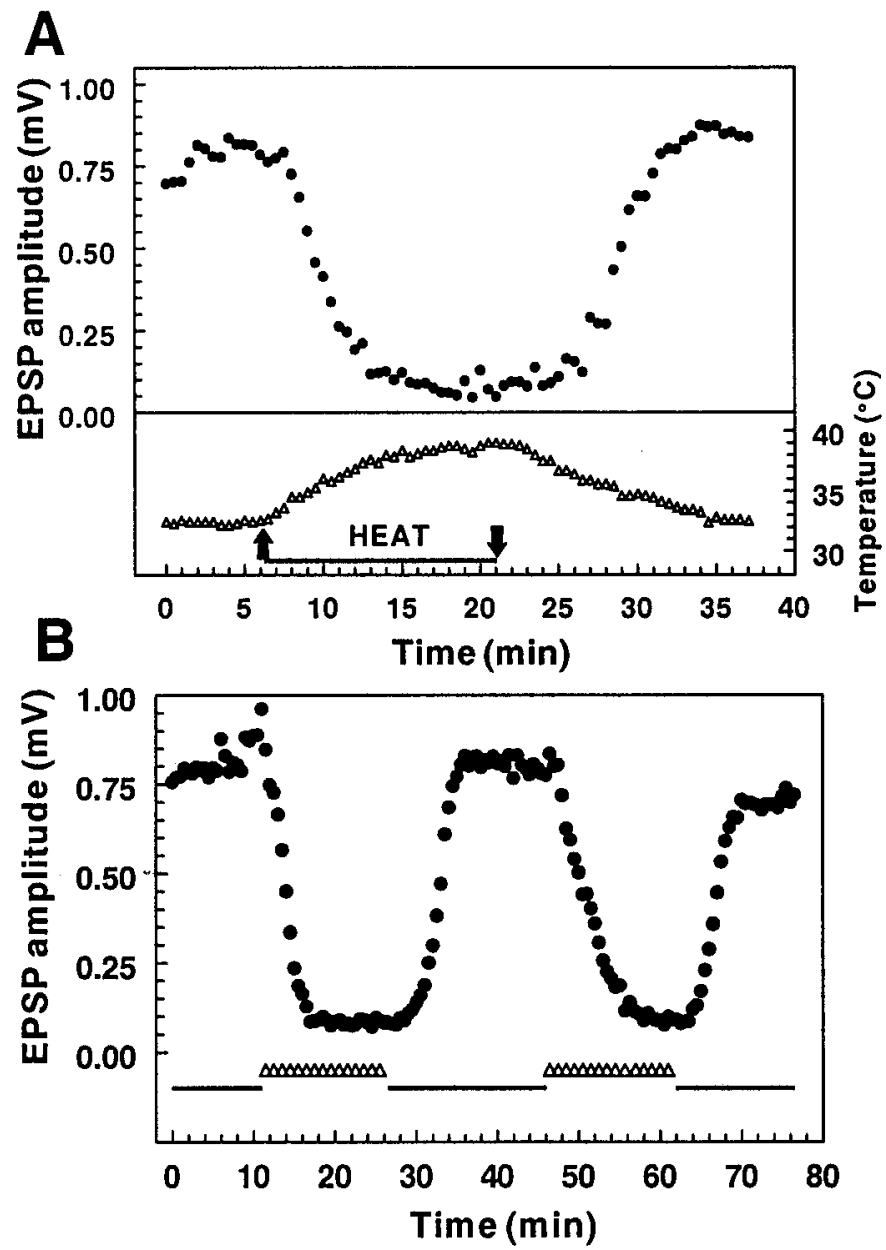

Figure 1. The effect of temperature on extracellular field potential amplitude. $A$, Changes in fEPSP amplitude (top) during and after an increase in the chamber temperature from $32.5 \rightarrow 38.5^{\circ} \mathrm{C}$ (bottom). Temperature changes were recorded with a small thermistor placed directly in the recording chamber along with the tissue slice. The peak amplitude of the fEPSP was inversely proportional to the temperature and showed full recovery to the baseline fEPSP amplitude when the temperature was returned to the initial recording temperature. $B$, The decrease in fEPSP amplitude in response to the temperature increase is repeatable in an individual slice. In this and all subsequent figures the baseline recording temperature $\left(32.5^{\circ} \mathrm{C}\right)$ is indicated by the solid line, and the transition to $38.5^{\circ} \mathrm{C}$, as illustrated in $A$, is indicated by the open triangles. The same temperature change illustrated in $A$ was executed twice in this slice, and a similar degree of synaptic inhibition was observed both times.

slices with this protocol, with the degree of inhibition ranging from as much as $90 \%$ to no change in the fEPSP. A sustained decrease in the fEPSP was observed in $80 \%$ (58 of 72) of hippocampal slices tested with the $32.5 \rightarrow 38.5^{\circ} \mathrm{C}$ paradigm; the remainder showed no change, a slight increase in the fEPSP, a transient decrease and spontaneous recovery, or a fluctuating response to the higher temperature. The $20 \%$ of slices that did not exhibit a characteristic decrease in the fEPSP in response to the temperature increase were not included in any additional characterization.

Figure $2 A$ shows the average response of 58 slices that responded to a transient temperature increase from $32.5 \rightarrow 38.5^{\circ} \mathrm{C}$, as illustrated in Figure 1. The fEPSP amplitude was decreased by $60.5 \pm 3.5 \%$ on warming and fully recovered to $100.7 \pm 4.6 \%$ of the initial baseline. There was no hysteresis in the temperature 

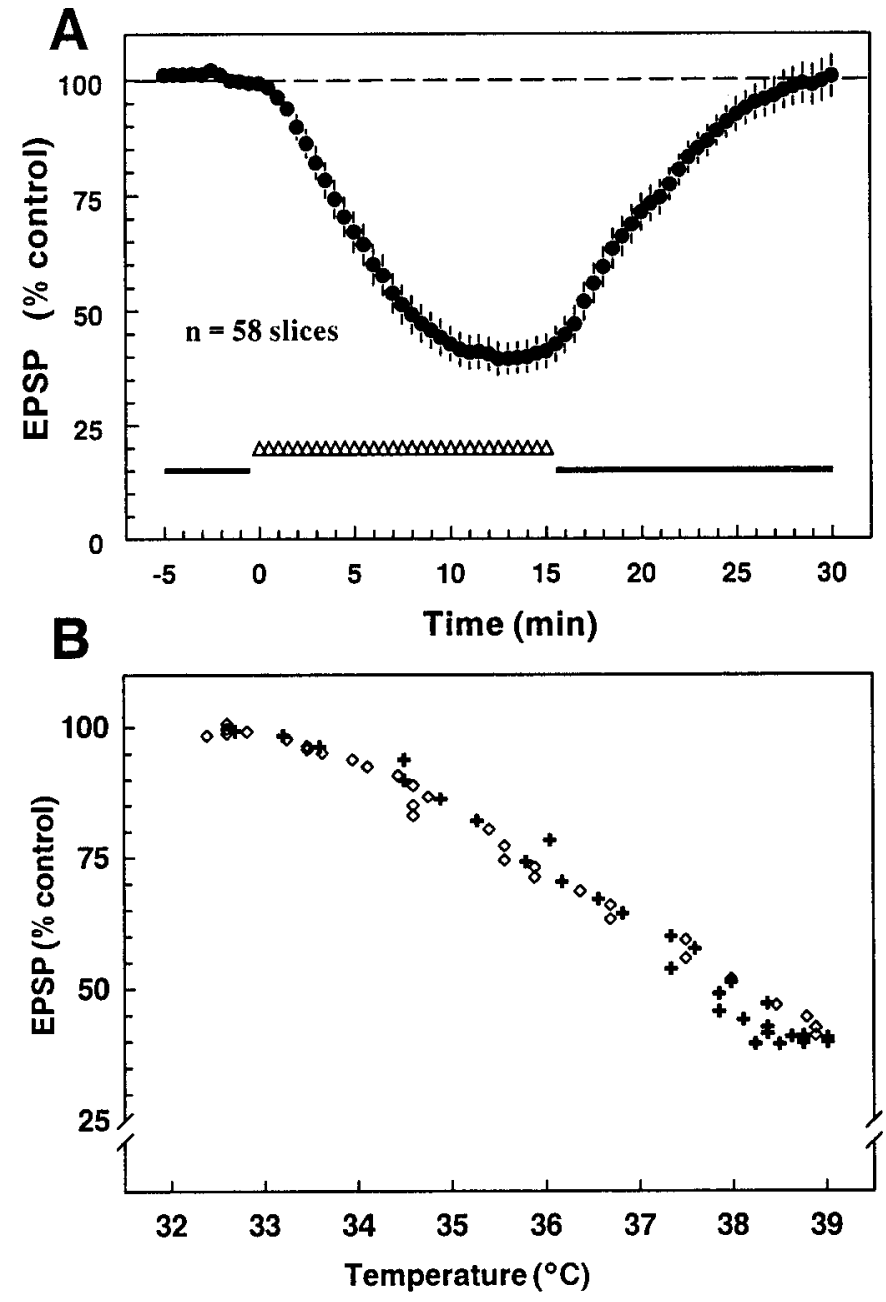

Figure 2. Effect of the $32.5 \rightarrow 38.5^{\circ} \mathrm{C}$ protocol on fEPSP amplitude. $A$, Shown is the time course of the mean response of 58 slices to the increase in temperature; each point is the mean \pm SEM for $80 \%$ of the slices tested with this protocol. The additional $20 \%$ not included in the average did not show the characteristic sustained fEPSP decrease in response to the increased temperature (the fEPSP increased slightly, exhibited no change, or oscillated in amplitude during the increased temperature). On average, the fEPSP amplitude was reduced to $39.5 \pm 3.5 \%$ of the control value by the temperature increase and recovered fully when the temperature change was reversed. $B$, Shown are the same data, but with the fEPSP plotted as a function of temperature instead of time. Responses elicited during the period when temperatures were increasing are indicated by a plus and during the decreasing phase by an open diamond. The relationship between temperature and response amplitude was nearly identical during both the temperature increase and decrease.

response, in that the relationship between the change in the fEPSP amplitude and temperature was nearly identical regardless of whether the temperature was being increased or decreased (Fig. 2B). The inhibition also did not appear to be dependent on the rate of change of the temperature, in that a similar degree of inhibition was observed either after a relatively slow change in temperature $\left(T_{1 / 2} \sim 5 \mathrm{~min}\right.$, as illustrated in Fig. $\left.1 A\right)$ or when the temperature was increased more rapidly $\left(T_{1 / 2}<2 \mathrm{~min}\right.$; data not shown). In addition, the synaptic inhibition did not occur at a specific threshold temperature, in that it could be elicited by increases from $32.5 \rightarrow 38.5^{\circ} \mathrm{C}$ as well as by increases from $\sim 23 \rightarrow 32^{\circ} \mathrm{C}$. In six of six slices that were tested with the $\sim 23 \rightarrow 32^{\circ} \mathrm{C}$ protocol, the EPSP initially increased on increasing the temperature (average increase, $58.0 \pm 7.5 \%$ ). Subsequent to this increase, three of six slices showed a decrease in the EPSP (average decrease measured from increased baseline, $46.0 \pm$ $14.7 \%$ ), similar to that seen with the $32.5 \rightarrow 38.5^{\circ} \mathrm{C}$ protocol, which also recovered when the temperature was decreased. Examples of synaptic responses recorded from individual slices before, during, and after the temperature increase from $32.5 \rightarrow 38.5^{\circ} \mathrm{C}$ are illustrated in Figure 3. In addition to the inhibition of the fEPSP (seen in Fig. $3 C$, trace $b, D$, trace $e$ ), there was also a decreased latency to the peak of the synaptic response, as would be expected because of faster conduction velocity as well as faster kinetics of the synaptic response at the higher temperature.

Because there did not appear to be an absolute temperature threshold for the effect, we determined if a relative threshold temperature increase was needed to produce significant inhibition. The change in temperature required to inhibit the fEPSP by $20 \%$ was determined for a population of slices incubated and recorded at $32.5^{\circ} \mathrm{C}$ and gradually raised to $38.5^{\circ} \mathrm{C}$ (Fig. 4). There was no clear-cut threshold for this effect; 12 of 58 slices showed a $20 \%$ inhibition with $\mathrm{a}<2^{\circ} \mathrm{C}$ change, but the thresholds ranged from $<2^{\circ} \mathrm{C}$ to $>5^{\circ} \mathrm{C}$ in others. There were three slices that exhibited a decrease, but that did not reach the $20 \%$ criterion with a $6^{\circ} \mathrm{C}$ increase tested. Although it was not investigated extensively, it also was noted that slices prepared from the same animal tended to respond more similarly than did slices from different animals, suggesting that minor differences in slice preparation, age, or other differences between individual animals may confer increased or decreased sensitivity to temperature changes.

\section{Mechanisms underlying the inhibitory response to temperature increases}

Because various other kinds of metabolic stresses, such as hypoxia and hypoglycemia (Fowler, 1993; Lloyd et al., 1993), increase the extracellular adenosine concentration in brain slices and because adenosine is a well characterized inhibitory presynaptic modulator at this synapse, we determined the effects of adenosine receptor antagonists on the response to temperature increases. Pretreatment with theophylline $(200 \mu \mathrm{M})$ antagonized the reduction in synaptic responses normally seen with the increased temperature (Fig. $5 A, C ; n=5 ; p<0.05$ ). Similarly, once maximal inhibition of the fEPSP was achieved during a period of increased temperature, theophylline was able to reverse the inhibition completely (Fig. $5 B ; n=4$ ). Because these experiments suggested that adenosine was responsible for the inhibition and presynaptic adenosine $A_{1}$ receptors have been linked to the inhibitory modulation of this synapse (Dunwiddie and Fredholm, 1989), the

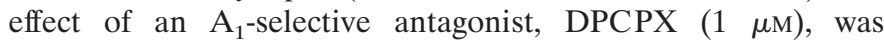
examined also. Pretreatment with DPCPX was as effective as theophylline in preventing the fEPSP decrease (Fig. $5 C ; n=4$; $p<0.005)$.

These results suggested that adenosine was most likely responsible for the synaptic inhibition induced by temperature increases. However, it was possible that another purine that is active at $A_{1}$ receptors might mediate the inhibitory response. To demonstrate that adenosine per se was responsible for the synaptic depression, we superfused slices with adenosine deaminase (ADA; 0.8-1.6 $\mathrm{U} / \mathrm{ml}$ ), which rapidly converts extracellular adenosine to inosine, which is essentially inactive at adenosine receptors. Pretreatment for 15 min before and during the temperature increase with this concentration of ADA significantly reduced the degree of inhibition when compared with the inhibition seen during the initial 

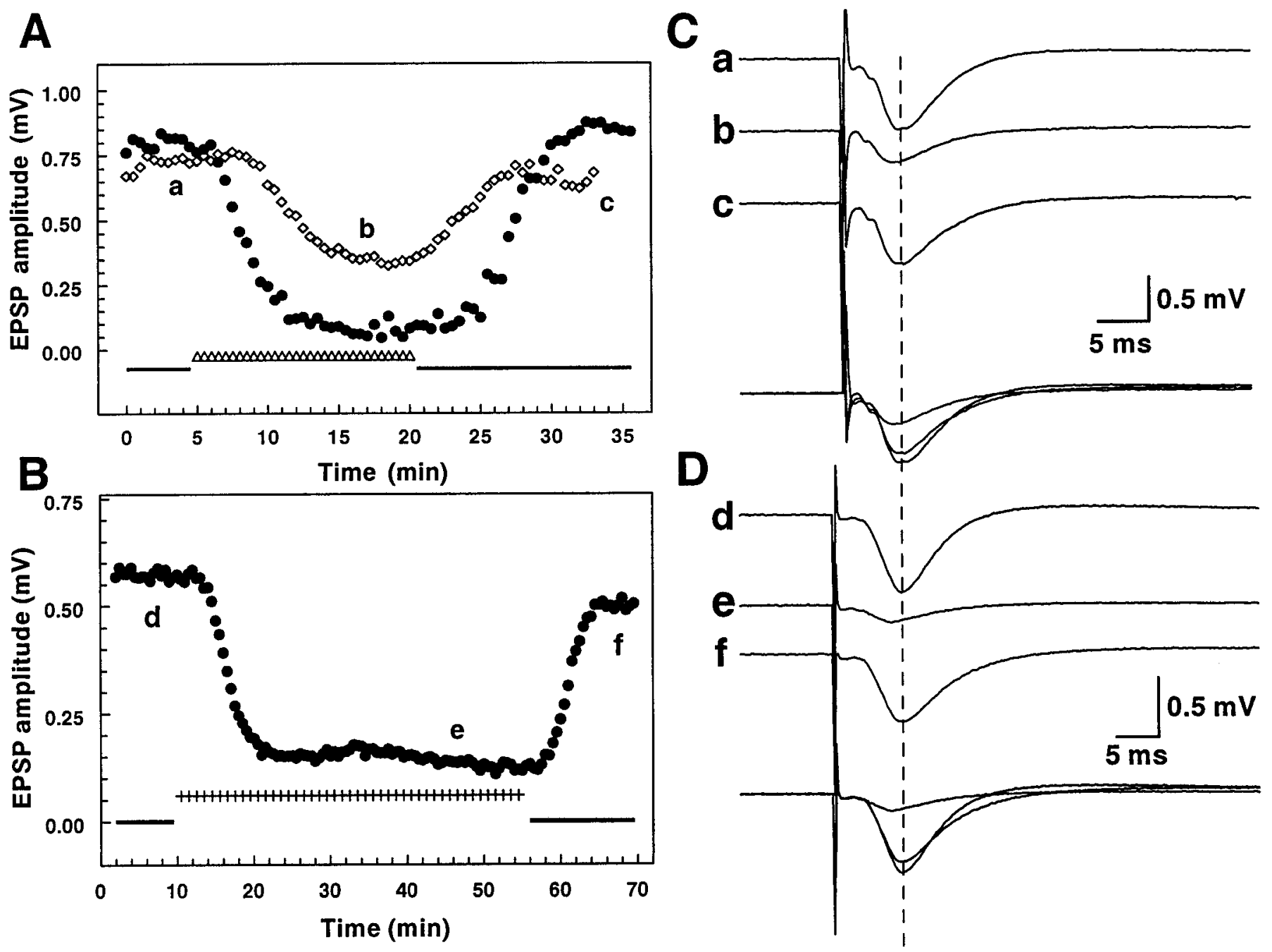

Figure 3. Responses of individual slices to changes in temperature. $A$, fEPSP amplitude changes in response to the standard temperature increase $\left(32.5 \rightarrow 38.5^{\circ} \mathrm{C}\right.$; the time course is illustrated in Fig. $\left.1 A\right)$. Although the temperature manipulation was identical, these two slices illustrate some of the variability in the latency, the degree of inhibition, and rate of fEPSP decrease in response to the temperature increase. $B$, Synaptic depression induced by an extended $32.5 \rightarrow 38.5^{\circ} \mathrm{C}$ protocol. In this slice the temperature increase was maintained for $45 \mathrm{~min}$; the inhibition of the fEPSP was maintained for the duration of the increased temperature and recovered when the recording temperature was returned to $32.5^{\circ} \mathrm{C}$. $C, D$, Examples of synaptic responses recorded from the hippocampal slices shown in $A$ and $B$; individual records correspond to responses evoked at the times indicated by the letters in $A$ $(a-c)$ and $B(d-f)$. The superimposed traces highlight the decreased latency to the peak of the fEPSP in traces $b$ and $e$ (peak fEPSP in baseline recordings $a$ and $d$ are indicated by a dashed vertical line), indicative of the faster kinetics of the response at the increased temperature.

temperature increase in the same set of slices (Fig. $5 C ; n=9$; $p<0.05)$.

\section{Mechanisms underlying increases in extracellular adenosine}

The preceding results point to an increased activation of $A_{1}$ receptors by endogenous adenosine as mediating the temperature-induced fEPSP decrease. There are multiple mechanisms that can lead to increases in extracellular adenosine, including efflux of cAMP from cells followed by extracellular conversion to adenosine (Rosenberg et al., 1994), direct efflux of adenosine itself (Lloyd et al., 1993), and an unknown mechanism that is initiated by activation of the NMDA receptors (Craig and White, 1992; Manzoni et al., 1994). NMDA receptor activation increases adenosine efflux from both cortical and hippocampal slices and is thought to result from the release of an unknown adenine nucleotide, followed by extracellular conversion to adenosine (Craig and White, 1993). However, application of neither the competitive NMDA receptor antagonist APV (50 $\mu \mathrm{M} ; n=3$, n.s.), nor the noncompetitive antagonist MK801 (10 $\mu \mathrm{M} ; n=11$, n.s.) before and during the temperature increase had any effect on the synaptic inhibition (Fig. $5 C$ ). Previous studies in other regions have suggested that activation of the NMDA receptors occurs even with relatively low rates of stimulation one stimulus every 20 sec; Harvey and Lacey, 1997) and is sufficient to initiate adenosine release. Therefore, experiments were conducted in which the synaptic stimulation was terminated before the temperature increase and then started at a time when the maximal change in the fEPSP would be expected. With this protocol the responses were inhibited maximally immediately on restarting the stimulation (decreased by $56.4 \% ; n=2$ ), providing further evidence that the activation of glutamate receptors by the synaptic stimulation was not involved in the increased concentrations of adenosine.

To evaluate the role of cAMP efflux as the source of extracellular adenosine, we inhibited the conversion of cAMP to $5^{\prime}$-AMP 


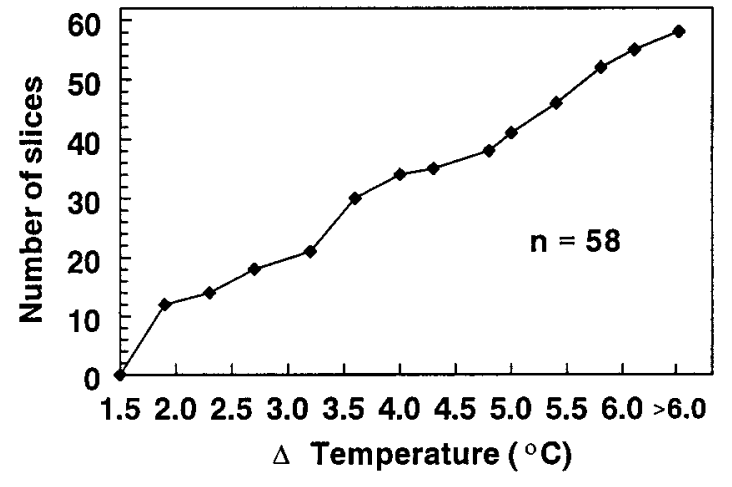

Figure 4. Temperature change needed to produce a $20 \%$ inhibition in the fEPSP with the $32.5 \rightarrow 38.5^{\circ} \mathrm{C}$ protocol. This cumulative histogram illustrates the temperature change required to produce a $20 \%$ decrease in fEPSP amplitude. Although a significant number of slices (12 of 58) exhibited this level of inhibition with a $<2^{\circ} \mathrm{C}$ increase in the recording temperature, others (17 of 58) did not respond with such a decrease until the temperature had changed by $>5^{\circ} \mathrm{C}$. The cumulative histogram was approximately linear over this range, indicating that there was a nearly uniform distribution of thresholds across this range. The median temperature change required to elicit a $20 \%$ decrease in the fEPSP was $3.4^{\circ} \mathrm{C}$.

(which subsequently can be dephosphorylated into adenosine) with the type IV phosphodiesterase inhibitor Ro 20-1724 (200 $\mu \mathrm{M}$; Bonci and Williams, 1996). In a separate set of experiments we blocked cAMP efflux directly by inhibiting the transporter that is primarily responsible for its release with probenecid (0.2-2 $\mathrm{mm})$. Neither Ro 20-1724 nor probenecid had a significant effect on the synaptic inhibition produced by the temperature increase (control inhibition vs Ro 20-1724 treatment, $80.4 \pm 10.9$ vs $70.4 \pm$ $20.9 \%$, respectively; $n=3$; control inhibition vs probenecid treatment, $53.3 \pm 10.4$ vs $58.9 \pm 11.4 \%$, respectively; $n=6$; both effects were nonsignificant by paired $t$ tests).

Because adenine nucleotides can be released and subsequently can be converted to adenosine via ectonucleotidases (Zimmerman, 1996), we evaluated the role of extracellular nucleotides as a source for extracellular adenosine. Slices were superf used with the ecto-ATPase inhibitor FPL $67156(200 \mu \mathrm{M})$ to prevent the conversion of extracellular ATP into ADP, which could undergo subsequent dephosphorylation into adenosine. We also used a combination of AOPCP and GMP (500 $\mu \mathrm{M}$ and $2 \mathrm{~mm}$, respectively) to inhibit the ecto-5' ${ }^{\prime}$-nucleotidase that converts AMP into adenosine. Neither treatment affected the synaptic inhibition produced by the temperature increase (control inhibition vs FPL 67156 treatment, $73.2 \pm 17.4$ vs $75.7 \pm 9.7 \%$, respectively; $n=2$; control inhibition vs AOPCP/GMP treatment, $52.2 \pm 9.3$ vs $32.1 \pm 9.7 \%$, respectively; $n=6$, n.s.). Although not significant, the AOPCP/GMP data showed a trend toward a reduced effect of temperature after pretreatment with this cocktail. Because superfusion with AOPCP/GMP resulted in a large decrease in the EPSP (most probably because of the inhibitory effect of AOPCP that has been noted by other groups as well; Cunha et al., 1998), the somewhat reduced response to the temperature increase might have been the consequence of a "floor" effect. To control for this, we performed three additional experiments in which the stimulus amplitude was increased after superfusion with AOPCP/ GMP to return the synaptic response to the baseline amplitude; under these conditions the inhibition in the presence of AOPCP/ GMP $(60.8 \pm 14.8 \%)$ was nearly identical to the control inhibition in the same slices $(60.2 \pm 13.8 \%)$. These results suggest that extracellular conversion of nucleotides is not the major source of adenosine in the current paradigm. However, with the inhibitors that are currently available, it is difficult to establish the complete inhibition of the ectoenzymes or phosphodiesterases targeted here, so these negative results should be interpreted with caution.

To determine the contribution of direct adenosine efflux as a source of extracellular adenosine, we used dipyridamole $(5 \mu \mathrm{M})$ to block the primary adenosine transporter found in the hippocampus (Deckert et al., 1988). Superfusion of dipyridamole had two effects on the synaptic inhibition consequent to the temperature increase. Dipyridamole increased the latency and decreased the rate of onset of the depression of synaptic responses (Fig. 6), suggesting that a dipyridamole-sensitive transporter mediates adenosine release during the temperature increase. If adenosine efflux were occurring via a nontransporterdependent mechanism, the rate of inhibition would be expected to increase, rather than decrease, because extracellular adenosine generated via such mechanisms would accumulate faster when uptake through the transporter is inhibited. Ultimately, dipyridamole did not reduce the maximal effect of the temperature increase; rather, it significantly augmented the extent of depression of the fEPSP response (control inhibition vs dipyridamole treatment, $60.2 \pm 10.3$ vs $83.6 \pm 1.6 \%$, respectively; $n=9 ; p<$ 0.05). This is likely attributable to dipyridamole blocking the usual role of this bidirectional nucleoside transporter in removing adenosine from the extracellular space; without the significant reuptake of adenosine, more adenosine accumulates under equilibrium conditions, although the rate of efflux may be reduced.

Because the metabolic energy requirements of tissue generally are increased at higher temperatures and because ischemia and hypoxia are well known stimulators of adenosine release, we considered the possibility that increases in temperature were making the slices hypoxic by increasing their metabolic rate. Previous studies with both hypoxia and ischemia have demonstrated that the ability of synaptic transmission to recover is a function of the duration of exposure and that prolonged treatment ( $>10 \mathrm{~min}$ and up to $1 \mathrm{hr}$, depending on the severity of the insult) leads to irreversible loss of, or a significant decrease in, subsequent synaptic transmission. Therefore, we determined the effects of maintaining slices for longer durations (45-65 $\mathrm{min}$ ) at increased temperatures on the magnitude of the fEPSP inhibition as well as the extent of subsequent recovery. When the temperature increase was maintained for a longer duration (see, e.g., Fig. $3 B$ ), the synaptic response remained depressed for the duration of the temperature increase and recovered after this extended temperature increase. In nine slices tested for $>45$ and up to $65 \mathrm{~min}$ with the $32.5^{\circ} \rightarrow 38.5^{\circ}$ protocol, the inhibition of the fEPSP was maintained throughout the increased temperature, but, more importantly, the fEPSP amplitude after recovery was nearly identical to the original baseline $(0.754 \pm 0.050 \mathrm{vs} 0.753 \pm 0.067 \mathrm{mV}$; $n=9$, n.s.). Finally, if the adenosine release were secondary to hypoxia/ischemia, the extent of recovery would be expected to be least complete in those slices that were most affected by the increased temperature. However, there was no correlation between the percentage of inhibition at increased temperature and the degree of recovery after the inhibition $(r=0.133$, n.s. $)$.

\section{DISCUSSION}

The results of this study demonstrate that increasing the temperature of hippocampal slices results in a profound decrease in the amplitude of synaptically evoked CA1 field potentials. The inhibition of synaptic transmission is fully reversible on cooling to the initial recording temperature, repeatable in individual slices, and 


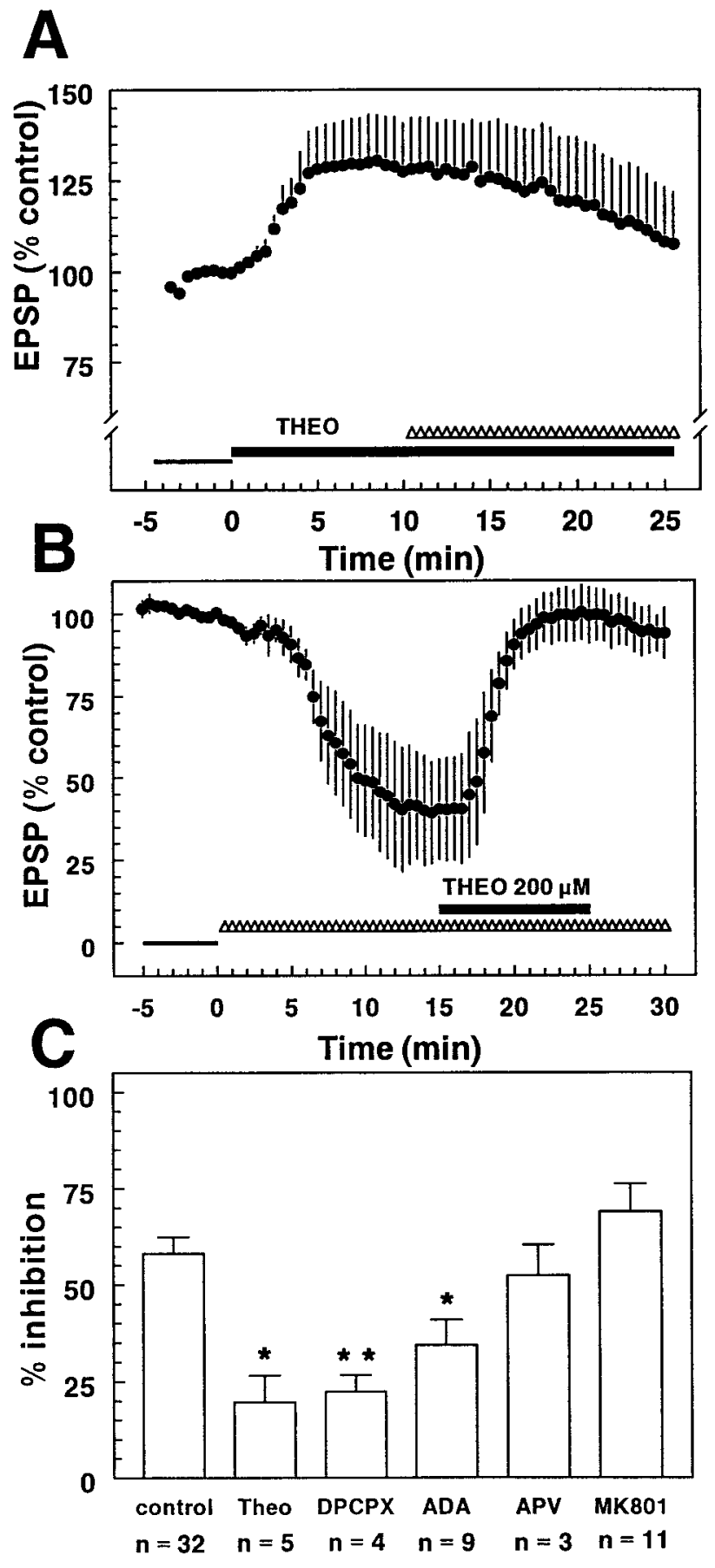

Figure 5. Effects of pharmacological antagonists on thermally induced synaptic depression $\left(32.5 \rightarrow 38.5^{\circ} \mathrm{C}\right)$. $A$, Pretreatment with theophylline (Theo; $200 \mu \mathrm{M}$ ) caused an initial increase in the fEPSP attributable to antagonism of the inhibitory effect of endogenous adenosine. Continued superfusion of theophylline during the temperature increase caused a significant reduction in the inhibition that typically is seen with the temperature increase (compare with Figs. 1-3). $B$, Theophylline not only reduced the depression of synaptic responses when it was superfused before a temperature increase $(A, B)$ but also fully reversed the effect of the temperature increase on the fEPSP amplitude once the depression had occurred $(n=4)$. $C$, In each slice the initial response to the increased temperature was recorded and compared with the effect of increased temperature in the same slice during drug superfusion (statistical comparisons were with paired Student's $t$ tests). The average control depression in all 32 slices tested with drugs is shown as control. The nonselective adenosine receptor antagonist theophylline (Theo; $200 \mu \mathrm{M})$, the adenosine $\mathrm{A}_{1}$ receptor antagonist DPCPX $(1 \mu \mathrm{M})$, and adenosine deaminase mediated primarily by adenosine $\mathrm{A}_{1}$ receptors. Increasing the temperature from 32.5 to $38.5^{\circ} \mathrm{C}$ produced a decrease in fEPSP amplitude in $80 \%$ of the slices that were tested. If similar changes in adenosine levels occur concurrently with endogenous temperature fluctuations in vivo, this suggests that there may be an important temperature dependency to the ongoing modulatory role of adenosine in the hippocampus and in the nervous system in general.

Given that normal endogenous fluctuations in brain temperature are somewhat less than the standard protocol used here, it is unclear whether these effects are relevant to more modest physiological temperature increases likely to occur in intact animals. Some slices did respond to temperature changes of $<2^{\circ} \mathrm{C}$, and, in vivo, body temperature changes of this magnitude can be initiated in a variety of ways, such as by increased physical activity, stress, or increased ambient temperature (Anderson et al., 1983; Kluger, 1991; Rothwell, 1994). However, all of the responses tested in the present experiments were from a hypothermic baseline (usually $32.5^{\circ} \mathrm{C}$ ), so it is unclear whether similar changes would ever be seen in vivo from a normothermic baseline. Although some studies provide support for the hypothesis that similar responses might occur in vivo [for example, Porkka-Heiskanen et al. (1997) have shown that brain adenosine levels are elevated at a time when circadian body temperatures are high (Refinetti and Menaker, 1992)], other studies suggest the opposite conclusion; (e.g., increased brain temperatures are associated with increased field potential amplitudes in vivo; Moser et al., 1993). Thus, future studies are required to establish the physiological relevance of this phenomenon.

Although the conditions studied here are not pathological, adenosine release has been established as a common way that cells respond to metabolically stressful conditions. The release of adenosine during cell stress has been shown to be neuroprotective, because adenosine receptor antagonists exacerbate neuronal cell loss initiated by hypoxia or ischemia (Rudolphi et al., 1987). Although adenosine release confers neuroprotection against pathological insults, its neuroprotective ability may be simply an extension of its more normal role in neuromodulation. Correlative evidence obtained in vivo suggests that an increase in body temperature is associated with an increase in brain adenosine levels. In many mammals the highest body temperature is found before sleep onset (Refinetti and Menaker, 1992), when extracellular adenosine in brain is highest (Porkka-Heiskanen et al., 1997). Extracellular adenosine levels in the basal forebrain increase during periods of sleep deprivation and drop during sleep (Porkka-Heiskanen et al., 1997), at which time the body temperature usually drops as well (Refinetti and Menaker, 1992). Similarly, Huston et al. (1996) demonstrated an increase in adenosine specific to the hippocampus as rats approach sleep, start to exhibit sleep-like behaviors, and decrease other behaviors. These researchers suggest that promotion of the restorative phase of sleep by adenosine may be related to the well characterized neuroprotective role of adenosine.

$\leftarrow$

$(A D A ; 0.8-1.6 \mathrm{U} / \mathrm{ml})$ all increased the baseline fEPSP (Theo, $136.2 \pm$ $15.1 \%$; DPCPX, $114.1 \pm 3.8 \%$; ADA, $122.9 \pm 12.8 \%)$, and all three significantly decreased the degree of synaptic depression as compared with that observed during an initial temperature increase $\left({ }^{*} p<0.05\right.$; $* * p<0.005)$. However, application of either the competitive NMDA receptor antagonist $\mathrm{APV}(50 \mu \mathrm{M})$ or the noncompetitive antagonist MK801 $(10 \mu \mathrm{M})$ had no significant effect on the synaptic depression. 


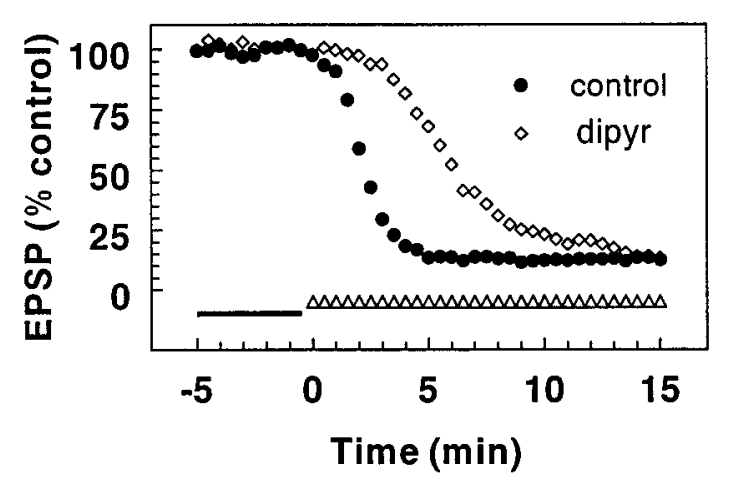

Figure 6. Effect of dipyridamole (dipyr) on latency of onset and rate of synaptic depression. Response amplitudes are shown for a single slice during an initial control temperature increase ( filled circles) and during a second temperature increase in which dipyridamole superfusion was begun immediately before the temperature increase (open diamonds). Dipyridamole delayed the onset of the depression and slowed the rate at which the synaptic response decreased during the temperature increase. Because dipyridamole alone results in a slow continuous decline in the fEPSP in most slices (Dunwiddie and Diao, 1994), statistical analyses of the time course of averaged responses were problematic, because most slices showed significant effects of dipyridamole alone. This individual slice was one in which the dipyridamole-induced decline was minimal at the time that the temperature was increased. Despite the possible additional effect of dipyridamole contributing to a decrease in the EPSP, there was instead a delay in the onset and a slowing of the rate of the inhibition.

In addition to altering overall metabolic rate, temperature changes are known to influence the dynamics of agonist and antagonist binding to receptors. With this in mind, we considered whether the inhibition observed here might be merely a result of an increased responsiveness to adenosine at higher temperatures rather than a change in adenosine concentrations. However, several studies suggest that this is not the case. Spangenberger et al. (1995) found that rat hippocampal slices were less sensitive to adenosine at increased temperatures and thus could not provide an explanation for our observations. In addition, although binding thermodynamics at $A_{1}$ receptors results in a small increase in agonist affinity with increased temperature, this effect reverses at temperatures above $35^{\circ} \mathrm{C}$ (Borea et al., 1995). Even if agonist affinity continued to increase linearly at temperatures above $35^{\circ} \mathrm{C}$, this could account only for $\sim 10 \%$ of the observed decrease in the fEPSP (Borea et al., 1991; Dunwiddie and Diao, 1994). Thus, changes in adenosine receptor sensitivity cannot account for the large synaptic depression observed in these experiments.

The mechanism underlying the adenosine increases observed in these studies is unclear. Although activation of the NMDA receptors is one potential mechanism that can result in an increase in extracellular adenosine in hippocampal slices (Manzoni et al., 1994), the lack of effect of NMDA receptor antagonists suggests that this mechanism was not involved. Adenosine concentrations appeared to change independently of direct electrical stimulation of the Schaffer collateral/commissural pathway, suggesting that postsynaptic glutamate receptor activation is not required. In addition, the potential role of adenine nucleotide release and its subsequent conversion to adenosine seems unlikely; however, the efficacy of inhibitors, particularly for $5^{\prime}$ nucleotidase, is somewhat problematic, so it is difficult to make definitive conclusions based on this kind of negative data. However, the delay in the onset of the inhibition with dipyridamole is consistent with the hypothesis that adenosine per se is released from cells within the brain slice. In biochemical studies the inhibitors of adenosine transport reduce the efflux of adenosine itself (Lloyd et al., 1993) because the transporters are essential for adenosine flux in either direction across cell membranes. The modest delay and slowed rate of onset of the hyperthermic response suggest that adenosine efflux through a dipyridamolesensitive transporter is the most likely source for the increase in extracellular adenosine.

Whatever mechanism accounts for the release of adenosine, the stimulus required is not hyperthermia per se, because the temperatures that were used were not hyperthermic; the average core temperature of a rat is between 37 and $38^{\circ} \mathrm{C}$ (Refinetti and Menaker, 1992), which were the highest temperatures tested. Moreover, we observed adenosine-mediated changes in synaptic transmission at temperatures that were in the hypothermic range $\left(23 \rightarrow 32^{\circ} \mathrm{C}\right)$. A recent paper by Gabriel et al. (1998) also demonstrated an adenosine-mediated synaptic depression in rat hippocampal slices increased from 22 to $29^{\circ} \mathrm{C}$. Taken together, this evidence emphasizes that the stimulus responsible for adenosine release is an increase in temperature but that hyperthermia itself is not involved.

Although the definitive mechanism underlying the temperaturerelated increase in extracellular adenosine is unclear, the results presented here have interesting implications for clinical as well as nonclinical situations. For instance, fever is associated with a general decrease in locomotor activity, an increase in sleep, and a reduced cognitive ability (Hart, 1988). Such symptoms are consistent with the known behavioral manifestations of increased extracellular adenosine and may be mediated at least in part via the increased activation of adenosine receptors. As noted, the endogenous circadian rhythm of body temperature and adenosine levels is consistent with the current results-both the highest body temperature and the highest adenosine concentrations are found before sleep onset (Refinetti and Menaker, 1992; PorkkaHeiskanen et al., 1997). It also should be noted that adenosine itself induces hypothermia and that stable $A_{1}$ receptor agonists can induce profound hypothermia in vivo (Dunwiddie and Worth, 1982). Finally, these results are relevant to brain slice studies, because changing temperatures even by several degrees may have significant functional consequences related to altered adenosine levels in the extracellular space.

Increased temperature may be similar to some pathological conditions in that it might be expected to produce an imbalance between metabolic requirements and the supply of oxygen and glucose to the tissue. However, in contrast to hypoxia or ischemia models, we observed full recovery of function even after $1 \mathrm{hr}$ at increased temperatures. Such recovery would not be possible consequent to these other insults, although the adenosine $A_{1}$ receptor-mediated synaptic inhibition is similar. Studies with mild to moderate hypoxia are perhaps most similar to those described here, insofar as the effects appear to be partially reversible even after $1 \mathrm{hr}$ (Arlinghaus and Lee, 1996). However, temperature changes sufficient to alter extracellular adenosine seem much more likely to occur both under normal physiological conditions and under more common conditions, such as during illness or exercise, than do other types of metabolic stress that have been shown to alter adenosine levels.

In conclusion, in this study we demonstrate that a mild form of stress-moderate temperature increase — causes a profound and sustained synaptic depression mediated by adenosine $A_{1}$ receptor activation in the CA1 region of the hippocampus. The characteristics of this synaptic depression include its full reversibility on cooling to the initial temperature and its repeatability in an 
individual slice. In this regard, an increase in temperature represents a nonpathological manipulation that markedly affects synaptic transmission via adenosine.

\section{REFERENCES}

Anderson RJ, Reed G, Knochel J (1983) Heatstroke. Adv Intern Med 28:115-140.

Arlinghaus L, Lee K (1996) Endogenous adenosine mediates the sustained inhibition of excitatory synaptic transmission during moderate hypoxia. Brain Res 724:265-268.

Barone FC, Feuerstein GZ, White RF (1997) Brain cooling during transient focal ischemia provides complete neuroprotection. Neurosci Biobehav Rev 21:31-44.

Berne RM, Rubio R, Curnish RR (1974) Release of adenosine from ischemic brain. Circ Res 35:262-271.

Bonci A, Williams JT (1996) A common mechanism mediates long-term changes in synaptic transmission after chronic cocaine and morphine. Neuron 16:631-639.

Borea PA, Varani K, Malaguti V, Gilli G (1991) Receptor binding at two different temperatures to discriminate agonist and antagonist behavior of adenosine $\mathrm{A}_{1}$ receptor ligands in rat brain. J Pharm Pharmacol 43:866-868.

Borea PA, Dalpiaz A, Guerra L, Gilli G (1995) Binding thermodynamics of adenosine $\mathrm{A}_{2 \mathrm{a}}$ receptor. Biochem Pharmacol 49:461-469.

Bruns RF, Lu GH, Pugsley TA (1986) Characterization of the $\mathrm{A}_{2}$ adenosine receptor labeled by $\left[{ }^{3} \mathrm{H}\right] \mathrm{NECA}$ in rat striatal membranes. Mol Pharmacol 29:331-346.

Craig CG, White TD (1992) Low-level $N$-methyl-D-aspartate receptor activation provides a purinergic inhibitory threshold against further $\mathrm{N}$-methyl-D-aspartate-mediated neurotransmission in the cortex. J Pharmacol Exp Ther 260:1278-1284.

Craig CG, White TD (1993) $N$-methyl-D-aspartate- and non- $N$-methylD-aspartate-evoked adenosine release from rat cortical slices: distinct purinergic sources and mechanisms of release. J Neurochem 60:1073-1080.

Cunha RA, Johansson B, Constantino MD, Sebastião AM, Fredholm BB (1996) Evidence for high-affinity binding sites for the adenosine $A_{2 A}$ receptor agonist $\left[{ }^{3} \mathrm{H}\right]$ CGS 21680 in the rat hippocampus and cerebral cortex that are different from striatal $\mathrm{A}_{2 \mathrm{~A}}$ receptors. Naunyn Schmiedebergs Arch Pharmacol 353:261-272.

Cunha RA, Sebastião AM, Ribeiro JA (1998) Inhibition by ATP of hippocampal synaptic transmission requires localized extracellular catabolism by ectonucleotidases into adenosine and channeling to adenosine $A_{1}$ receptors. J Neurosci 18:1987-1995.

Deckert J, Bisserbe JC, Klein E, Marangos PJ (1988) Adenosine uptake sites in brain: regional distribution of putative subtypes in relationship to adenosine $\mathrm{A}_{1}$-receptors. J Neurosci 8:2338-2349.

Dunwiddie TV (1985) The physiological role of adenosine in the central nervous system. Int Rev Neurobiol 27:63-139.

Dunwiddie TV, Diao L (1994) Extracellular adenosine concentrations in hippocampal brain slices and the tonic inhibitory modulation of evoked excitatory responses. J Pharmacol Exp Ther 268:537-545.

Dunwiddie TV, Fredholm BB (1989) Adenosine $A_{1}$ receptors inhibit adenylate cyclase activity and neurotransmitter release and hyperpolarize pyramidal neurons in rat hippocampus. J Pharmacol Exp Ther 249:31-37.

Dunwiddie TV, Hoffer BJ (1980) Adenine nucleotides and synaptic transmission in the in vitro rat hippocampus. Br J Pharmacol 69:59-68.

Dunwiddie TV, Lynch G (1978) Long-term potentiation and depression of synaptic responses in the rat hippocampus: localization and frequency dependency. J Physiol (Lond) 276:353-367.

Dunwiddie TV, Worth T (1982) Sedative and anticonvulsant effects of adenosine analogs in mouse and rat. J Pharmacol Exp Ther 220:70-76.

Flagmeyer I, Haas HL, Stevens DR (1997) Adenosine $A_{1}$ receptormediated depression of corticostriatal and thalamostriatal glutamatergic synaptic potentials in vitro. Brain Res 778:178-185.
Fowler JC (1993) Purine release and inhibition of synaptic transmission during hypoxia and hypoglycaemia in rat hippocampal slices. Neurosci Lett 157:83-86.

Gabriel A, Klussmann FW, Igelmund P (1998) Rapid temperature changes induce adenosine-mediated depression of synaptic transmission in hippocampal slices from rats (non-hibernators) but not in slices from golden hamsters (hibernators). Neuroscience 86:67-77.

Hart BL (1988) Biological basis of the behavior of sick animals. Neurosci Biobehav Rev 12:123-137.

Harvey J, Lacey MG (1997) A postsynaptic interaction between dopamine $\mathrm{D}_{1}$ and NMDA receptors promotes presynaptic inhibition in the rat nucleus accumbens via adenosine release. J Neurosci 17:5271-5280.

Huston JP, Haas HL, Boix F, Pfister M, Decking U, Schrader J, Schwarting RKW (1996) Extracellular adenosine levels in neostriatum and hippocampus during rest and activity periods of rats. Neuroscience 73:99-107.

Kluger MJ (1991) Fever: role of pyrogens and cryogens. Physiol Rev 71:93-127.

Lloyd HGE, Lindström K, Fredholm BB (1993) Intracellular formation and release of adenosine from rat hippocampal slices evoked by electrical stimulation and energy depletion. Neurochem Int 23:173-185.

Manzoni OJ, Manabe T, Nicoll RA (1994) Release of adenosine by activation of NMDA receptors in the hippocampus. Science 265:2098-2101.

Moser E, Mathiesen I, Anderson P (1993) Association between brain temperature and dentate field potentials in exploring and swimming rats. Science 259:1324-1326.

Motley SJ, Collins GGS (1983) Endogenous adenosine inhibits excitatory transmission in the rat olfactory cortex slice. Neuropharmacology 22:1081-1086.

Porkka-Heiskanen T, Strecker R, Thakkar M, Bjørkum AA, Greene R, McCarley RW (1997) Adenosine: a mediator of the sleep-inducing effects of prolonged wakefulness. Science 276:1265-1268.

Refinetti R, Menaker M (1992) The circadian rhythm of body temperature. Physiol Behav 51:613-637.

Rosenberg PA, Knowles R, Knowles KP, Li Y (1994) $\beta$-Adrenergic receptor-mediated regulation of extracellular adenosine in cerebral cortex in culture. J Neurosci 14:2953-2965.

Rothwell NJ (1994) CNS regulation of thermogenesis. Crit Rev Neurobiol 8:1-10.

Rubio R, Berne RM, Bockman EL, Curnish RR (1975) Relationship between adenosine concentration and oxygen supply in rat brain. Am J Physiol 228:1896-1902.

Rudolphi KA, Keil M, Hinze HJ (1987) Effect of theophylline on ischemically induced hippocampal damage in Mongolian gerbils: a behavioral and histopathological study. J Cereb Blood Flow Metab 7:74-81.

Spangenberger H, Nikmanesh FG, Igelmund P (1995) Effects of adenosine on synaptic transmission in hippocampal slices from hibernating and warm-acclimated Turkish hamsters and rats. Neurosci Lett 185:217-219.

Svenningsson P, Nomikos GG, Ongini E, Fredholm BB (1997) Antagonism of adenosine $\mathrm{A}_{2 \mathrm{~A}}$ receptors underlies the behavioural activating effect of caffeine and is associated with reduced expression of messenger RNA for NGFI-A and NGFI-B in caudate-putamen and hippocampus. Neuroscience 79:753-764.

Tanimoto M, Okada Y (1987) The protective effect of hypothermia on hippocampal slices from the guinea pig during deprivation of oxygen and glucose. Brain Res 417:239-246.

Zhu PJ, Krnjević K (1993) Adenosine release is a major cause of failure of synaptic transmission during hypoglycaemia in rat hippocampal slices. Neurosci Lett 155:128-131.

Zhu PJ, Krnjević K (1997) Adenosine release mediates cyanide-induced suppression of CA1 neuronal activity. J Neurosci 17:2355-2364.

Zimmerman H (1996) Extracellular purine metabolism. Drug Dev Res 39:337-352 